\title{
Cathepsin $L$ derived from skeletal muscle cells transfected with bFGF promotes endothelial cell migration
}

\author{
Ji Hyung Chung ${ }^{1,2,3,4,7}$, Eun Kyoung Im $^{1,3}$, \\ Taewon Jin ${ }^{1,3,4}$, Seung-Min Lee ${ }^{5}$, \\ Soo Hyuk Kim ${ }^{1,3,4}$, Eun Young Choi ${ }^{1,3,4}$, \\ Min-Jeong Shin ${ }^{5}$, Kyung Hye Lee ${ }^{1,3,4}$ \\ and Yangsoo Jang ${ }^{1,2,3,6}$ \\ ${ }^{1}$ Cardiovascular Research Institute \\ ${ }^{2}$ Cardiovascular Product Evaluation Center \\ Yonsei University College of Medicine \\ Seoul 120-752, Korea \\ ${ }^{3}$ Yonsei Research Institute of Science for Aging \\ Yonsei University \\ Seoul 120-749, Korea \\ ${ }^{4}$ Severance Integrative Research Institute for \\ Cerebral and Cardiovascular Diseases \\ Yonsei University Health System \\ Seoul 120-752, Korea \\ ${ }^{5}$ Institute of Health Science \& Department of Food and Nutrition \\ College of Health Science \\ Korea University \\ Seoul 136-703, Korea \\ ${ }^{6}$ Severance Medical Research Institute \\ Yonsei University Health System \\ Seoul 120-752, Korea \\ ${ }^{7}$ Corresponding author: Tel, +82-2-2228-0374; \\ Fax, +82-2-2227-7906; E-mail, jhchung@ yonsei.ac.kr \\ DOI 10.3858/emm.2011.43.4.022
}

Accepted 24 February 2011

Available Online 24 February 2011

Abbreviations: bFGF, basic fibroblast growth factor; HUVECs, human umbilical artery endothelial cells; JNK, c-Jun N-terminal kinase; MMP-1, matrix metalloproteinase-1; PAI-1, plasminogen activator inhibitor-1; SkMCs, skeletal muscle cells; t-PA, tissue-type plasminogen activator; uPA, urokinase plasminogen activator; VEGF, vascular endothelial growth factor

\begin{abstract}
Gene transfer of basic fibroblast growth factor (bFGF) has been shown to induce significant endothelial migration and angiogenesis in ischemic disease models. Here, we investigate what factors are secreted from skeletal muscle cells (SkMCs) transfected with bFGF gene and whether they participate in endothelial cell migration. We constructed replication-defective ad-
\end{abstract}

enovirus vectors containing the human $b F G F$ gene (Ad/bFGF) or a control LacZ gene (Ad/LacZ) and obtained conditioned media, bFGF-CM and LacZ-CM, from SkMCs infected by $\mathrm{Ad} / \mathrm{bFGF}$ or $\mathrm{Ad} / \mathrm{LacZ}$, respectively. Cell migration significantly increased in HUVECs incubated with bFGF-CM compared to cells incubated with LacZ-CM. Interestingly, HUVEC migration in response to bFGF-CM was only partially blocked by the addition of bFGF-neutralizing antibody, suggesting that bFGF-CM contains other factors that stimulate endothelial cell migration. Several proteins, matrix metalloproteinase-1 (MMP-1), plasminogen activator inhibitor-1 (PAI-1), and cathepsin L, increased in bFGF-CM compared to LacZ-CM; based on 1-dimensional gel electrophoresis and mass spectrometry. Their increased mRNA and protein levels were confirmed by RT-PCR and immunoblot analysis. The recombinant human bFGF protein induced MMP-1, PAl-1, and cathepsin L expression in SkMCs. Endothelial cell migration was reduced in groups treated with bFGF-CM containing neutralizing antibodies against MMP-1 or PAl-1. In particular, HUVECs treated with bFGF-CM containing cell-impermeable cathepsin $L$ inhibitor showed the most significant decrease in cell migration. Cathepsin L protein directly promotes endothelial cell migration through the JNK pathway. These results indicate that cathepsin $L$ released from SkMCs transfected with the $b F G F$ gene can promote endothelial cell migration.

Keywords: fibroblast growth factor 2; cathepsin L; endothelium; JNK Mitogen-Activated Protein Kinases; cell migration

\section{Introduction}

Angiogenesis is the biological process of growing new blood vessels from preexisting vessels, which involves the disruption of the extracellular matrix (ECM) by proteolytic enzymes, endothelial cell proliferation and migration, and the subsequent formation and maturation of new capillary tubes (Markkanen et al., 2005; Chen et al., 2009). Endothelial cell migration is essential to angiogenesis, which is regulated by chemotactic, haptotactic, and mechanotactic stimuli (Lamalice et al., 2007). Chemotactic endothelial cell migration is asso- 
ciated with several growth factors, such as vascular endothelial growth factor (VEGF), bFGF, and other cytokines (Lamalice et al., 2007; Chen et al., 2010; Kim et al., 2010). Although several growth factors contribute to proliferative and migratory events, bFGF plays a key role in endothelial cell migration through its cognate receptor and intracellular signaling response (Pintucci et al., 2002). Also, bFGF has a wide spectrum of target cells, including smooth muscle cells, fibroblasts, keratinocytes, and SkMCs involved in diverse biological responses (Barrientos et al., 2008), suggesting that endothelial cell migration is probably regulated by communication between the endothelium and the surrounding environment.

Proteases are also involved in normal physiological processes, including angiogenesis and vasculogenesis (van Hinsbergh et al., 2006). Among them, three groups of endoproteases are known to play a crucial role in the regulatory pathways that control various processes of angiogenesis such as endothelial cell migration, invasion, and remodeling of the extracellular matrix. These endoproteases comprise the zinc-dependent MMPs, the serine proteases such as UPA, and cathepsin cysteine proteases. It has been extensively studied that MMPs are the key molecules associated with tissue remodeling and wound healing response as well as cell migration and invasion (Le et al., 2007). Serine proteases such as UPA and plasmin have also been reported to modulate cellular adhesion and migration in various physiological conditions (D'Alessio and Blasi, 2009). Another family of proteases consists of the cysteine cathepsins which are expressed in the cell surface and secreted into the extracellular space (Palermo and Joyce, 2008). Cathepsins have recently emerged as an important class of proteolytic enzymes in cell migration and invasion. Cathepsin L, in particular, has broad substrate specificity and activates several molecules involved in angiogenesis (Stoka et al., 2007). Cathepsin L induces the ECM degradation (Gocheva and Joyce, 2007) and stimulates the recruitment and action of blood-derived accessory cells that enhance angiogenesis (van Hinsbergh et al., 2006).

Because gene delivery using growth factors has been shown to induce beneficial effects in animal models (Yla-Herttuala, 2006), angiogenic gene therapy can be a reasonable strategy to achieve the same effects. The therapeutic potential of bFGF gene delivery has also been confirmed in myocardial and limb ischemia models (Ninomiya et al., 2003; Kondoh et al., 2004). In most cases of therapeutic gene delivery, an expression vector harboring target genes is injected intramuscularly, and the exogenous gene is expressed mainly in the muscle cells. In the in vivo angiogenic gene transfer approach, a foreign gene is expressed in non-endothelial cells and the angiogenic process is regulated by the participation of multiple factors from several cells. Therefore, the paracrine actions of other factors secreted from non-endothelial cells together with the direct effects of the therapeutic gene seem to be a sound mechanism to explain the improved functional outcomes in therapeutic angiogenesis trials. In previous studies using limb ischemia animal models, intramuscular injections of the bFGF gene showed increased expression of hepatocyte growth factor (Onimaru et al., 2002) and placenta growth factor (Fujii et al., 2008). This suggests another mechanism for the increase of angiogenesis with $b F G F$ gene transfer is the secretion of several factors from non-endothelial cells, including SkMCs. However, little is known about the expression of growth factors and cytokines stimulated by bFGF in skeletal muscle, which is a target tissue of gene delivery for limb diseases. Thus, we sought to identify novel factors secreted from SkMCs transfected with $b F G F$ that contribute to endothelial cell migration in vitro. In the present study, we tested what factors are secreted from SkMCs with $\mathrm{Ad} /$ $b F G F$ transfection and whether they participate in endothelial cell migration associated with angiogenesis.

\section{Results}

\section{bFGF expression in skeletal muscle cells}

Human SkMCs were infected with a replicationdefective adenoviral vector (Ad/bFGF) containing the human $b F G F$ gene. After $72 \mathrm{~h}$, the level of bFGF expression was evaluated by reverse transcriptase-polymerase chain reaction (RT-PCR). The bFGF expression from the Ad/bFGF-infected cells was significantly higher than the LacZ genecontaining adenoviral vector (Ad/LacZ)-infected cells or the blank group (uninfected cells) (Figure $1 A)$. Secretion of $b F G F$ protein from Ad/bFGFinfected cells was detected by immunoblot analysis. The level of bFGF protein was significantly greater in SkMC media infected with $\mathrm{Ad} / \mathrm{bFGF}$ than in SkMC media infected with Ad/LacZ (Figure 1B). These results demonstrate that a recombinant adenoviral vector harboring the $b F G F$ gene could successfully transfer into cells and efficiently produce the bFGF protein in SkMCs. The amount of bFGF protein secreted from Ad/bFGF-infected cells increased in a time-dependent manner (Figure 1C). 
A

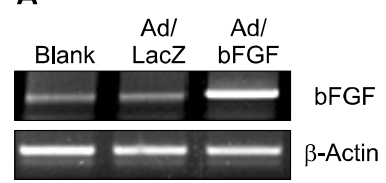

C

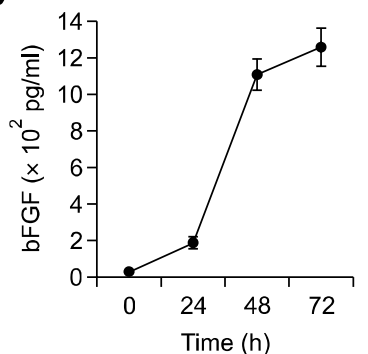

Figure 1. bFGF expression and secretion in the SkMCs infected with Ad/bFGF. (A) SkMCs were transfected with Ad/LacZ or Ad/bFGF. After $72 \mathrm{~h}$, total RNA was isolated from SkMCs and RT-PCR was performed. (B) The culture media from cells trasfected with Ad/LacZ or Ad/bFGF and uninfected cells (Blank) were collected. Equal amounts of protein were separated by SDS-PAGE, and bFGF protein was detected by immunoblot analysis using anti-bFGF antibody. (C) The amount of bFGF secreted from cultured SkMCs was measured with the human bFGF ELISA kit. The results represent the means \pm SEM of five different experiments.

\section{Effect of bFGF-conditioned SkMC medium on endothelial cell migration}

We examined the effect of bFGF-CM collected from SkMCs infected with $A d / b F G F$ on endothelial cell migration. The effect of bFGF-CM on endothelial cell migration was determined by Boyden chamber migration assay. When HUVECs were incubated with bFGF-CM ( $50 \%$ in basal medium), cell migration significantly increased compared to cells incubated with LacZ-conditioned medium (LacZ-CM, 50\% in basal medium) (Figure 2A). To determine whether this significant increase can be attributed exclusively to the effect of bFGF protein in bFGF-CM, we analyzed endothelial migration using a bFGF-neutralizing antibody. The addition of exogenous bFGF protein $(2 \mathrm{ng} / \mathrm{ml})$ to basal culture medium accelerated cell migration and the addition of bFGF-neutralizing antibody completely prevented endothelial cell migration (Figure 2B). However, HUVEC migration in response to bFGF-CM was only partially blocked by the addition of a bFGFneutralizing antibody (Figure 2B). The bFGF-CMinduced HUVEC migration was not totally inhibited even at higher concentrations of the bFGFneutralizing antibody (more than $10 \mu \mathrm{g} / \mathrm{ml}$ ) (data not shown). The addition of a control IgG antibody did not change the cell migration of the bFGF protein-treated group or bFGF-CM-treated group (data not shown). From these data, we infer that
A
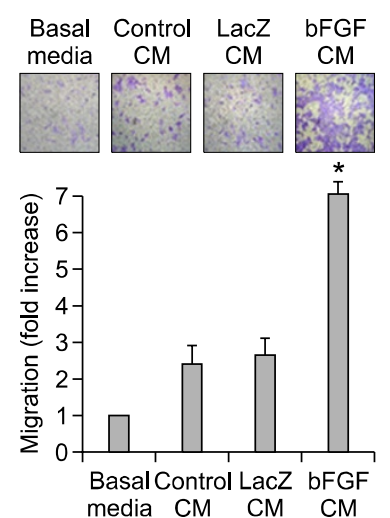

B

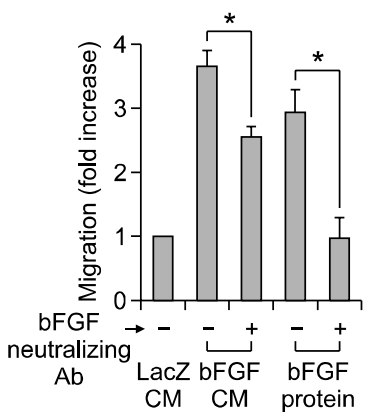

Figure 2. Effect of bFGF-CM on HUVECs migration. (A) HUVEC migration was stimulated by addition of basal media, conditioned medium from uninfected SkMCs (Control CM), conditioned medium from SkMCs transfected with Ad/LacZ (LacZ-CM) or conditioned medium from SkMCs transfected with Ad/bFGF (bFGF-CM). After $12 \mathrm{~h}$, cells that migrated to the other side of the membrane in Boyden chamber were stained with $1 \%$ crystal violet and eluted with methanol, and quantitative analyses were performed by optical density. The results represent the means \pm SEM of three different experiments. ${ }^{*} P<0.01$ compared to LacZ-CM group. (B) HUVEC migration was stimulated by addition of LacZ-CM, bFGF-CM or bFGF protein (2 $\mathrm{ng} / \mathrm{ml}$, in basal media). The bFGF-neutralizing antibody $(3 \mu \mathrm{g} / \mathrm{ml})$ was co-treated to the chamber. After $12 \mathrm{~h}$, migrated cells were stained and quantified. The results represent the means \pm SEM of three different experiments. ${ }^{*} P<0.01$.

bFGF-CM contains other factors, in addition to bFGF, that stimulate endothelial cell migration.

\section{Identification of factors in bFGF-CM of SkMCs}

We decided to identify other factors besides bFGF in bFGF-CM using a proteomic strategy. To identify endothelial migration factors secreted from SkMCs infected with $A d / b F G F$, both conditioned media (LacZ-CM or bFGF-CM) were harvested and concentrated, and proteins were analyzed by $8-16 \%$ one-dimensional gradient SDS-PAGE and silver staining. Individual protein bands were excised from the stained gel and digested by trypsin, and the resulting peptides were analyzed by mass spectrometer (Supplementary Figure 1). As expected, $\beta$-galactosidase and bFGF bands were found in LacZ-CM and bFGF-CM, respectively (Figure 3A). Our analysis revealed that several proteins, MMP-1, PAI-1, cathepsin L, moesin and cyclophilin B, increased in bFGF-CM compared to LacZ-CM (Figure $3 A$ ). No protein was identified in a 90-kDa band. In order to quantitatively confirm the changes of in expression level, mRNA levels of several factors identified by mass spectrometry were measured using RT-PCR. The mRNA levels of three factors, MMP-1, PAI-1, and cathepsin L, were remarkably increased in SkMCs infected with Ad/bFGF com- 
A

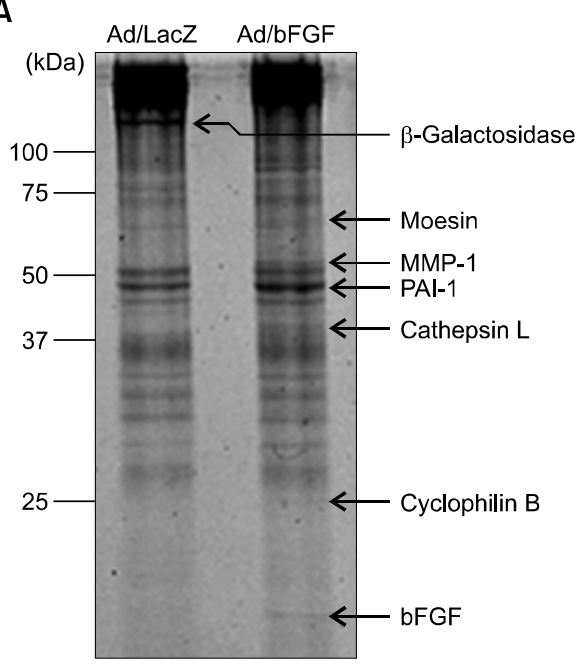

C

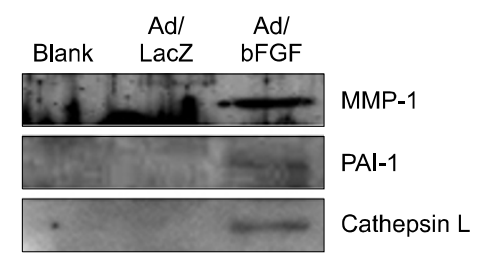

B

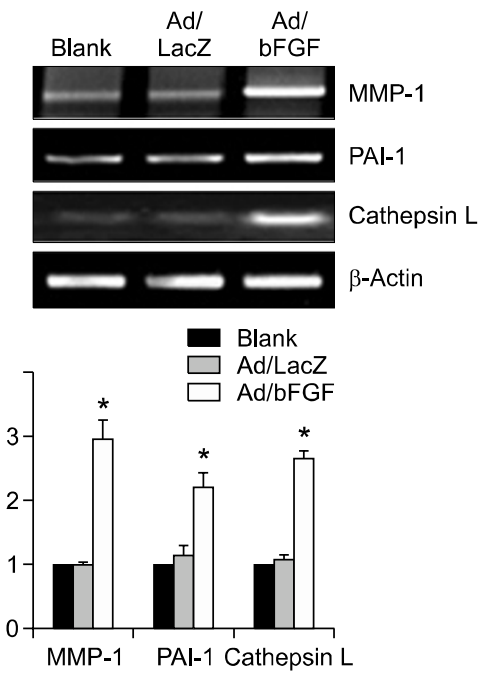

Figure 3. Analysis of factors secreted from SkMCs transfected with bFGF. (A) SkMCs were transfected with $\mathrm{Ad} / L a c Z$ or Ad/bFGF. After $72 \mathrm{~h}$, each conditioned media were concentrated ten times, and secreted proteins were analyzed by $8-16 \%$ gradient SDS-PAGE. Proteins separated by SDS-PAGE were subjected to in-gel digestion by trypsin and analyzed by MALDI-TOF mass spectrometry. (B) SkMCs were transfected with Ad/LacZ or Ad/bFGF. After $72 \mathrm{~h}$, total RNA was isolated from SkMCs and RT-PCR was performed. The PCR products were electrophoresed on $1.5 \%$ agarose gel. Bands were quantified by densitometry, and the values were normalized to that of $\beta$-actin mRNA. Blank, RT-PCR band from uninfected cells. ${ }^{*} P<0.01$ compared to the blank (uninfected cells). (C) SkMCs were transfected with Ad/LacZ or Ad/bFGF. After $72 \mathrm{~h}$, culture media were collected and concentrated ten times. Equal amounts of protein were separated by SDS-PAGE and analyzed by immunoblot assay.

pared to $A d / L a c Z$-infected cells (Figure $3 B$ ). The protein levels were the same in the culture medium (bFGF-CM) of SkMCs infected with Ad/bFGF (Figure $3 \mathrm{C}$ ). There was little difference in the mRNA and protein levels of other factors (moesin and cyclophilin B) between the $\mathrm{Ad} / \mathrm{LacZ}$ and $\mathrm{Ad} /$ $b F G F$ groups (data not shown).

\section{bFGF induces expression of three factors in SkMCs}

The expression of three factors in SkMCs infected with $\mathrm{Ad} / \mathrm{bFGF}$ suggests they were released by the autocrine effect of bFGF in response to the bFGF gene transfer into the SkMCs. To test this hypothesis, SkMCs were stimulated with bFGF protein and the mRNA level in the cells and the protein level in the media were measured by RT-PCR and ELISA, respectively. As shown in Figure 4A, recombinant human bFGF protein induced the expression of MMP-1, PAl-1 and cathepsin L. Corresponding to the mRNA levels, the bFGF protein treatment significantly increased the amount of these factors in cell culture media (Figure 4B). Hence, these results suggest that the production of these factors may result from the autocrine effect in response to the bFGF released from SkMCs transfected with bFGF gene.

\section{Cathepsin L in bFGF-CM of SkMC is critical for endothelial cell migration}

To determine whether these factors released from the bFGF-CM of SkMC contribute to endothelial cell migration, we examined the migration of HUVECs by treating them with bFGF-CM containing neutralizing antibodies or a chemical inhibitor. Cell migration was significantly reduced when the HUVECs were treated with bFGF-CM containing MMP-1 neutralizing antibody (Figure 5A) compared to treatment with $\mathrm{bFGF-CM}$ alone. Cell migration also slightly decreased in the PAI-1 neutralizing antibody-treated bFGF-CM group. In particular, 
A

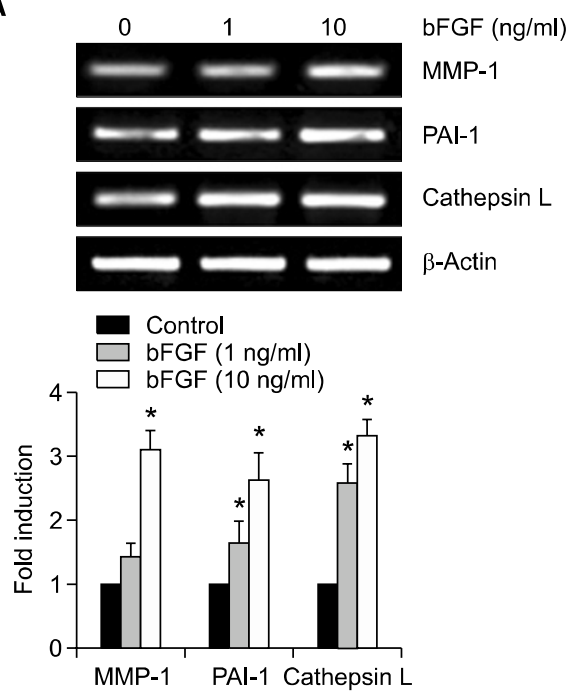

B

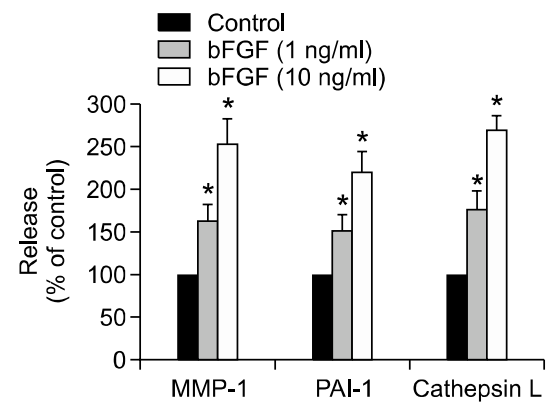

Figure 4. Expression and secretion of MMP-1, PAl-1 and cathepsin L by bFGF protein treatment in SkMCs. (A) SkMCs were treated with bFGF protein (1 or $10 \mathrm{ng} / \mathrm{ml})$. After $24 \mathrm{~h}$, total RNA was isolated from SkMCs and RT-PCR was performed. The PCR products were electrophoresed on $1.5 \%$ agarose gel. Bands were quantified by densitometry, and the values were normalized to that of $\beta$-actin mRNA. ${ }^{*} P<0.01$ compared to the control. (B) The amount of MMP-1, PAl-1 and cathepsin L secreted from cultured SkMCs was measured using ELISA kits. The results represent the means \pm SEM of five different experiments. ${ }^{*} P<0.01$ compared to the control.

HUVECs treated with bFGF-CM containing cellimpermeable cathepsin $\mathrm{L}$ inhibitor (Z-Phe-Tyr-CHO) showed the most significant decrease in cell migration (Figure $5 \mathrm{~A}$ ); this occurred in a concentrationdependent manner (Figure 5B).

\section{Cathepsin $L$ induces endothelial cell migration via c-Jun N-terminal kinase (JNK)}

Next, we studied whether indeed the three factors directly stimulate endothelial cell migration. The HUVECs were exposed to MMP-1, PAI-1, or cathepsin $\mathrm{L}$ for $12 \mathrm{~h}$ and a Boyden chamber assay was performed. We observed that MMP-1 treatment resulted in an increase of HUVEC migration in a concentration-dependent manner (Figure 6A). Only a slight increase in cell migration was found after treatment with PAI-1. Of the three factors, cathepsin $L$ is the most effective in stimulating HUVEC migration; compared to the control, it stimulated endothelial cell migration from 2.2- to 3.9 -fold at concentrations of 10 and $100 \mathrm{ng} / \mathrm{ml}$, respectively (Figure 6A). To characterize the intracellular signaling pathways that mediate the effects of cathepsin $L$ on endothelial cell migration, we focused on MAP kinases because of their involvement in regulating cell migration in several cell types, including endothelial cells. We found that cathepsin $\mathrm{L}$ induced phosphorylation of JNK in HUVECs in a concentration-dependent manner (Figure 6B). The protein levels of total JNK were unchanged for all concentrations of cathepsin $L$ (data not shown). We next examined whether a JNK-specific inhibitor could prevent cathepsin Linduced endothelial cell migration. As shown in Figure 6C, treatment with the JNK-specific inhibitor,
A

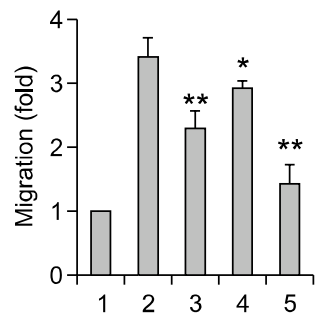

B

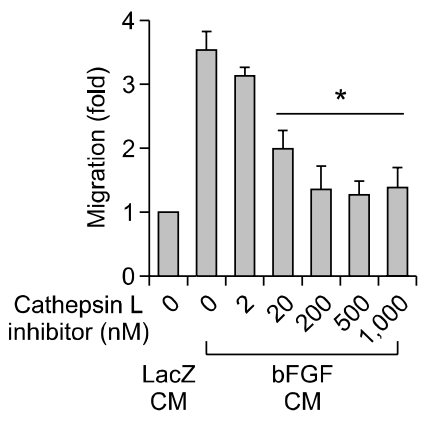

Figure 5. Effect of cathepsin $L$ inhibitor on the bFGF-CM-induced HUVECs migration. (A) HUVECs were incubated with $b F G F-C M$ alone or medium containing MMP-1 neutralizing antibody $(5 \mu \mathrm{g} / \mathrm{ml})$, PAl-1 neutralizing antibody $(5 \mu \mathrm{g} / \mathrm{ml})$, or cathepsin L inhibitor $(200 \mathrm{nM})$ for $12 \mathrm{~h}$. Migrated cells were stained and quantified. The results represent the means \pm SEM of three different experiments. 1, LacZ-CM; 2, bFGF-CM; 3, bFGF-CM + MMP-1 neutralizing Ab (5 $\mu \mathrm{g} / \mathrm{ml}) ; 4$, bFGF-CM + PAl-1 neutralizing $\mathrm{Ab}(5 \mu \mathrm{g} / \mathrm{ml}) ; 5, \mathrm{bFGF}-\mathrm{CM}+$ Cathepsin $\mathrm{L}$ inhibitor $(200 \mathrm{nM})$. ${ }^{*} P<0.05$ compared to the bFGF-CM group. ${ }^{*} P<0.01$ compared to the bFGF-CM group. (B) HUVECs were incubated with bFGF-CM containing different concentration of cathepsin L inhibitor in Boyden chamber for $12 \mathrm{~h}$. Cell migration assay was also performed in the same way as above. The results represent the means \pm SEM of three different experiments. ${ }^{*} P<0.01$ compared to the bFGF-CM group. 
A

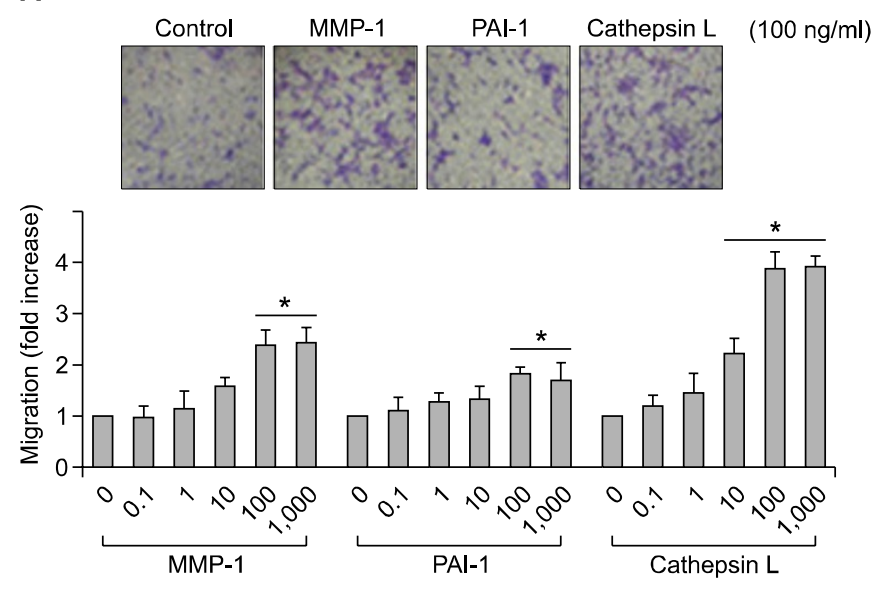

B

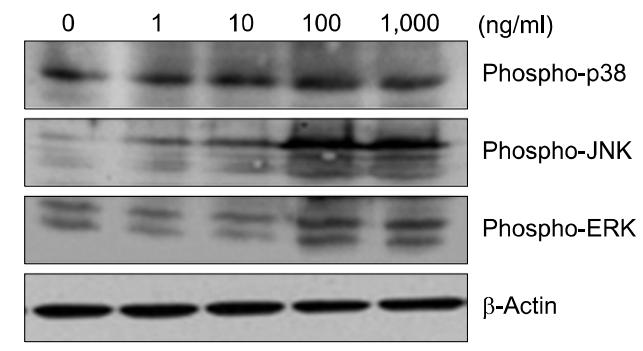

C

Control

Cathepsin L

Cathepsin L + SP600125

SP600125
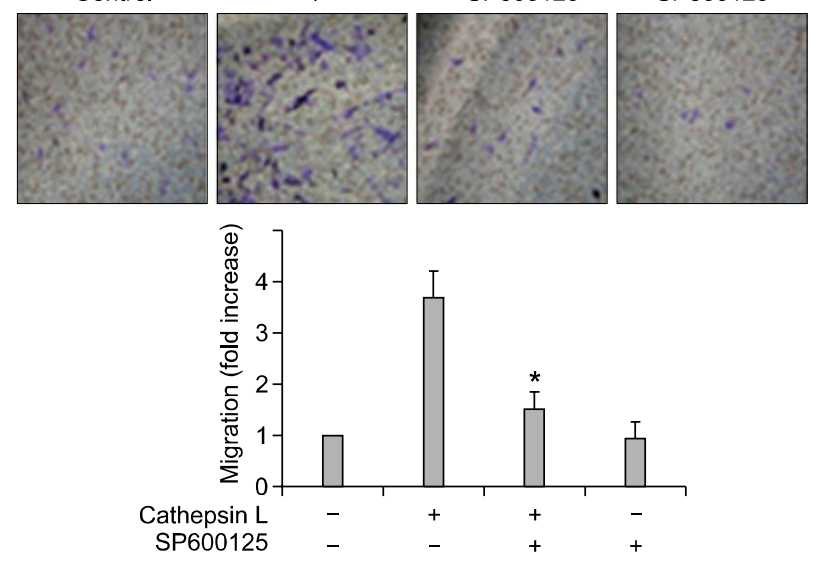

Figure 6. Effect of cathepsin L on HUVEC migration. (A) HUVEC migration was stimulated by addition of MMP-1, PAl-1 or cathepsin L. After $12 \mathrm{~h}$, migrated cells were stained and quantified. The results represent the means \pm SEM of three different experiments. ${ }^{*} P<0.01$ compared to the control. (B) HUVECs were treated with various concentrations of cathepsin L for 30 min. Equal amounts of protein were separated by SDS-PAGE gel, and immunoblot analysis was performed. (C) HUVECs were pretreated with a JNK inhibitor, SP600125 (25 $\mu \mathrm{M})$, for $1 \mathrm{~h}$. Then cell migration was stimulated by addition of cathepsin $L(100 \mathrm{ng} / \mathrm{ml})$. After $12 \mathrm{~h}$, migration assay was performed in the same way as above $(A)$. The results represent the means $\pm S E M$ of three different experiments. ${ }^{*} P<0.01$ compared to the cathepsin $L$ group.

SP600125, significantly inhibited the cell migration induced by cathepsin L in HUVECs.

\section{Discussion}

Several studies have revealed the roles of bFGF in angiogenesis, including endothelial cell proliferation and migration. In response to bFGF, a large number of cellular signaling processes occur in order to regulate cellular events, including cell proliferation and migration (Turner and Grose, 2010). The bFGF has also proved to be a promising therapeutic agent in treating myocardial and hindlimb ischemia (Ninomiya et al., 2003; Nishikage et al., 2004). Most in vivo studies have reported that $b F G F$ gene delivery improves the functional outcome in ischemic models; however, the focus has only been on the autocrine effect of bFGF in endothelial cells. Because, in the case of in vivo gene transfer for ischemic limb diseases, intramuscular injection of a viral vector or plasmid DNA is used for gene delivery to an intact skeletal muscle, paracrine factors released from these tissues may play an important role in endothelial cell proliferation and migration.

The results of the present study provide important evidence in support of roles for factors released from SkMCs transfected with the bFGF gene in the regulation of endothelial cell migration. The main finding of this study is that mere bFGF accumulation in the bFGF-CM is not sufficient to induce 
endothelial migration. We found that bFGF-CM from SkMCs significantly induced HUVEC migration, which was reduced when a bFGF-neutralizing antibody was added to the conditioned media. However, it is important to note that cell migration did not completely decline to the control level in the bFGF antibody-treated group, indicating that other secreted factors in the bFGF-CM also affect endothelial cell migration. We identified several proteins secreted from SkMCs in response to bFGF stimulation using 1-D gel electrophoresis and mass spectrometry. The increased production of three factors, MMP-1, PAI-1, and cathepsin L, in the bFGF-CM of SkMCs cells was confirmed by RTPCR and immunoblot analysis (Figure 3). Because bFGF exerts its effects through its transmembrane receptor, production of these factors may result from the autocrine effect in response to bFGF released from SkMCs transfected with the $b F G F$ gene. Indeed, a marked enhancement of mRNA expression and protein levels was observed in SkMCs treated with bFGF protein (Figure 4). The induction of these three factors by Ad/bFGF infection significantly decreased in the presence of the bFGF neutralizing antibody in SkMCs (Supplementary Figure 2).

One of the factors identified in our study is MMP-1, a zinc-dependent proteolytic enzyme involved in the remodeling of the extracellular matrix and the proteolytic processing of interstitial collagenase for the degradation of connective tissue (Pardo and Selman, 2005). MMP-1 contributes to physiological processes, such as tissue repair, regeneration, and invasion (Pulukuri and Rao, 2008) and it also directly promotes tube formation of endothelial cells. This suggests MMP-1 is associated with pro-angiogenic events (Blackburn and Brinckerhoff, 2008). Research regarding the effects of bFGF on MMP-1 expression has demonstrated that bFGF administration increase MMP-1 expression in human periodontal ligament cells (Palmon et al., 2000). An increase of MMP-1 protein was also observed when bFGF was added to human skin fibroblasts (Xie et al., 2008). Our study shows that overexpression of bFGF leads to upregulation of MMP-1 in SkMCs and that stimulation of SkMCs with bFGF protein also results in increased MMP-1 expression and secretion. In addition, we have demonstrated that MMP-1 from bFGF-conditioned media plays an important role in endothelial cell migration (Figure 5A). Its effect on cell migration was confirmed by treating HUVECs with MMP-1 protein (Figure 6A), indicating direct a role for endothelial cell migration and supporting important roles such as cell invasion and matrix degradation in angiogenesis.
PAl-1, a regulator of both tissue-type plasminogen activator (t-PA) and urokinase plasminogen activator (UPA), has also been shown to play a multifunctional role in various processes associated with vascular remodeling (Nicholl et al., 2006; Binder et al., 2007). PAI-1 also plays a crucial role in the cell adhesion and migration of vascular cells and in angiogenesis (Agirbasli, 2005; Binder et al., 2007). However, controversial data about the proangiogenic and antiangiogenic effects of PAI-1 have been described. It is likely that PAl-1 functions depending on the experimental conditions and interactions with different molecules. In fact, the angiogenic response of PAI-1 was shown to depend on its concentration. In other words, PAI-1 is proangiogenic at physiological concentrations and antiangiogenic at higher levels (Devy et al., 2002). But, bFGF definitely increased PAl-1 mRNA and the accumulation of PAI-1 protein in vascular endothelial cells (Kaneko et al., 2002) and smooth muscle cells (Watanabe et al., 2001). Our data show that PAl-1 is secreted from SkMCs transfected with $b F G F$, and it influences the migration of HUVECs. The induction of UPA was also observed in SkMCs treated with bFGF (Supplementary Figure 3). These results in turn could account, at least in part, for the stimulatory effect of the uPA-PAI-1 complex in bFGF-induced endothelial migration.

Of particular interest in this study is the role of cathepsin $L$ in endothelial cell migration. The involvement of PAI-1, UPA, and MMPs in endothelial migration and angiogenesis is documented, but a possible role of cathepsin $L$ has not yet been addressed. Cathepsin L, a lysosomal protease, plays an important role in intracellular protein degradation. It is secreted from the cell under certain conditions (Collette et al., 2004). Cathepsin $\mathrm{L}$ has also been shown to contribute to tumor cell invasion (Yang and Cox, 2007), metastasis (Lah et al., 2006), and malignancy (Skrzydlewska et al., 2005). Urbich et al. have reported that cathepsin $L$ has a critical role in vasculogenesis by promoting the invasive activity of endothelial progenitor cells. Moreover, they showed that transfection with cathepsin $L$ resulted in an increased invasive capacity in HUVECs (Urbich et al., 2005). The identification of cathepsin $L$ as a stimulator of endothelial cell migration is interesting from a biological point of view. Above all, bFGF induces significant increases in cathepsin $L$ in SkMCs (Figure 3, Supplementary Figure 4), and most importantly the cathepsin L effect is required for bFGF-induced endothelial cell migration. Another finding of the present study is that endothelial cell migration by cathepsin $L$ is mediated through the 
JNK pathway (Figure 6, Supplementary Figure 5). Since the direct role of cathepsin $L$ on endothelial cells and its action mechanism have not been well demonstrated, our study may encourage the consideration of a novel function of this protease. We cannot rule out the possibility of the association of cathepsin $\mathrm{L}$ with other proteins in the regulating endothelial migration because cathepsin $\mathrm{L}$ can generate potent antiangiogenic molecule, such as endostatin (Felbor et al., 2000). Therefore, further studies will be necessary to investigate how cathepsin $L$ production is regulated in response to bFGF and whether cathepsin $L$ also affects the expression of other proteins in endothelial cells. In summary, we have shown that several factors are secreted from bFGF-overexpressed SkMCs, which promote endothelial cell migration. In particular, we report here, for the first time, that cathepsin $L$ plays a direct and crucial role in endothelial cell migration and that this effect is mediated through the activation of the JNK pathway.

\section{Methods}

\section{Materials}

Anti-human bFGF antibody and bFGF-neutralizing antibody were purchased from Millipore (Bedford, MA). AntiMMP-1 antibody was purchased from Chemicon (Temecula, CA). Anti-PAI-1 and anti-cathepsin L antibodies were obtained from Santa Cruz Biotechnology (Santa Cruz, CA). Anti-phospho-p38, anti-phospho-JNK, and anti-phospho-ERK antibodies were purchased from Cell Signaling (Beverly, MA). Recombinant MMP-1 protein and bFGF protein were purchased from R\&D Systems (Minneapolis, MN) and Invitrogen (Camarillo, CA), respectively. PAl-1 and cathepsin L proteins were purchased from Calbiochem (San Diego, CA). Neutralizing antibodies against MMP-1 and PAl-1, and cell-impermeable cathepsin L inhibitor (cathepsin inhibitor II, Z-Phe-Tyr-CHO) were also purchased from Calbiochem. Human MMP-1 and cathepsin L ELISA kits were from R\&D Systems. Human PAI-1 ELISA kit was obtained from Abfrontier (Seoul, Korea). SB203580 and PD98059 were purchased from AG Scientific (San Diego, CA). SP600125 was procured from Calbiochem.

\section{Cell culture}

SkMCs (Lonza, Walkersville, MD) were cultured in SKGM (Lonza) with supplements on a $5 \% \mathrm{CO}_{2}$ atmosphere at $37^{\circ} \mathrm{C}$. HUVECs (Lonza) were maintained in EBM-2 medium (Lonza) supplemented with $2 \%$ fetal calf serum (FCS), according to manufacturer's instructions. For all experiments, cells were used up to the ten passages and harvested at $90 \%$ confluence.

\section{Preparation of adenoviral vector}

A recombinant cDNA for the secreted form of human bFGF was constructed by adenovirus expression vector kit (Takara Bio, Shiga, Japan). The bFGF cDNA was placed into a cosmid vector ( $p A x C A w t)$ which contains potent CAG promoter and nearly the full-length adenovirus genome deficient E1 and E3 genes. This cosmid vector inserted with $b F G F$ DNA and DNA-TPC digested with a restriction endonuclease EcoT22I (Takara Bio) were co-transfected into 293 cells. Recombinant adenovirus was generated by homologous recombination according to manufacturer's instructions. The recombinant adenovirus $\mathrm{Ad} / \mathrm{bFGF}$ was purified by ultra-centrifugation through a $\mathrm{CsCl}_{2}$ gradient followed by extensive dialysis. The titer of the virus stock was assessed by a plaque-formation assay using the 293 cells and expressed in plaque formation units. The control adenovirus $\mathrm{Ad} / \mathrm{LacZ}$ expressing bacterial $\beta$-galactosidase was also constructed by same method.

\section{Preparation of conditioned media}

Human SkMCs were grown in SKGM in the presence of $10 \%$ fetal bovine serum (FBS) until $80-90 \%$ confluence. The growth medium was then removed, cells were incubated in basal media (SKBM, Lonza) containing $0.1 \%$ BSA for $16 \mathrm{~h}$. Cells were then infected with Ad/bFGF (10 $\mathrm{moi}$ ) or $\mathrm{Ad} / \mathrm{LacZ}$. After $6 \mathrm{~h}$, cells were washed twice with SKBM and incubated at $37^{\circ} \mathrm{C}$ in a humidified atmosphere of $5 \% \mathrm{CO}_{2}$ for $72 \mathrm{~h}$. Conditioned media from Ad/bFGFinfected cells (bFGF-CM), Ad/LacZ-infected cells (LacZ$\mathrm{CM}$ ) or uninfected cells (control-CM) were then collected, centrifuged for $10 \mathrm{~min}$ at $3,000 \times g$ to remove cell debris and stored at $-80^{\circ} \mathrm{C}$. The amount of secreted bFGF in the conditioned medium was measured with the human bFGF ELISA kit (R\&D Systems), according to the manufacturer's instructions.

\section{Cell migration assay}

HUVECs $\left(1 \times 10^{5}\right.$ cells) were seeded into $8.0-\mu \mathrm{m}$ pore transwell inserts (Corning, Corning, NY). Inserts containing HUVECs were placed into a 24-well plate containing basal medium (EBM-2) with $0.2 \%$ FBS. HUVECs migration was stimulated by addition of $1 \mathrm{ml}$ of $5 \times$ conditioned media (bFGF-CM or LacZ-CM) or $2 \mathrm{ng} / \mathrm{ml}$ of bFGF protein to the lower well of the Boyden chamber. After $12 \mathrm{~h}$, the surface of the upper membrane was swabbed with a cotton-tipped applicator to remove non-migrating cells. Inserts were fixed in methanol for 30 min and stained with $1 \%$ crystal violet for $2 \mathrm{~h}$. For quantitative analysis, the surface of membrane was eluted by methanol and optical density was measured using a microplate reader.

\section{RT-PCR}

Total RNA from SkMCs was isolated using the RNA extraction reagent Trizol (Invitrogen). RNA samples were reverse-transcribed using oligo-dT primer and Moloney murine leukemia virus reverse transcriptase (Gibco BRL, Grand Island, NY) at $42^{\circ} \mathrm{C}$ for $2 \mathrm{~h}$. Then the CDNAs were amplified by PCR in the presence of each specific primer and Taq DNA polymerase. The cDNA fragments corresponding to bFGF, MMP-1, PAI-1 and cathepsin $L$ were amplified with the following sets of primers: 5'-GAGGA 
GTTGTGTCTATCAAAG-3' and 5'-GTTCGTTTCAGTGCCA CATACC-3' for bFGF, 5'-GGATTGAAAATTACACG-3' and 5'CTTTCATCTTCATCAAAATG-3' for MMP-1, 5'-CAGACCA AGAGCCTCTCCAC-3' and 5'-ATCACTTGGCCCATGAA AAG-3' for PAl-1, 5'-GTCAGTGTGGTTCTTGTTGG-3' and 5'-AAGGACTCATGACCTGCATC-3' for cathepsin L, and 5'CGTGGGCCGCCCTAGGCACCA-3' and 5'-TTGGCCTTA GGGTTCAGGGGGG-3' for $\beta$-actin. The PCR products were electrophoresed on $1.5 \%$ agarose gels. Each DNA band was visualized by staining with ethidium bromide and quantified by densitometer using TINA-image analysis program.

\section{SDS-PAGE and immunoblot analysis}

Cell lysates containing equal amounts of protein were subjected to SDS-polyacrylamide gel electrophoresis, and the proteins transferred to polyvinylidene difluoride (PVDF) membranes. After blocking in $10 \mathrm{mM}$ Tris-Cl buffer ( $\mathrm{pH} 8.0)$ containing $150 \mathrm{mM}$ sodium chloride, $0.1 \%$ Tween 20 , and $5 \%(\mathrm{w} / \mathrm{v})$ non-fat dry milk, the membranes were treated with primary antibodies, followed by appropriate horseradish peroxidase-conjugated secondary antibodies. The antigen-antibody bands were detected with a Western Blotting Luminol Reagent kit (Santa Cruz Biotechnology) following manufacturer's instruction.

\section{One-dimensional gel electrophoresis and silver staining}

Conditioned media were collected and concentrated ten times, and proteins were electrophoresed through an 8$16 \%$ gradient polyacrylamide precast gel (Bio-Rad). The gel was then stained using Silver Stain Plus kit (Bio-Rad). The gel was first fixed in a fixative enhancer solution $(50 \%$ methanol, $10 \%$ acetic acid and $10 \%$ fixative enhancer concentrate) for $20 \mathrm{~min}$. The gel was then washed twice in deionized distilled water for $10 \mathrm{~min}$. The Gel was incubated in the staining solution (5\% sliver complex solution, $5 \%$ reduction moderator solution, $5 \%$ image development reagent and $50 \%$ development accelerator solution) until proteins were visible.

\section{Mass spectrometry and protein identification}

Individual protein bands were excised, chopped into 1-mm² pieces. Proteins were subjected to in-gel trypsin digestion. Excised gel spots were destained with $100 \mathrm{ml}$ of destaining solution $(50 \% \mathrm{MeOH}$ in $10 \%$ acetic acid) with shaking for 5 min. After removal of the solution, gel spots were incubated with $200 \mathrm{mM}$ ammonium bicarbonate for $20 \mathrm{~min}$. The gel pieces were dehydrated with $100 \mathrm{ml}$ of acetonitrile and dried in a vacuum centrifuge. The gel pieces were rehydrated with $20 \mathrm{ml}$ of $50 \mathrm{mM}$ ammonium bicarbonate containing $0.2 \mathrm{mg}$ modified trypsin (Promega, Madison, $\mathrm{WI}$ ) for $45 \mathrm{~min}$ on ice. After removal of solution, $30 \mathrm{ml}$ of 50 $\mathrm{mM}$ ammonium bicarbonate was added. The digestion was performed overnight at $37^{\circ} \mathrm{C}$. The peptide solution was desalted using C18 nano column (home-made). Custommade chromatographic columns were used for desalting and concentration of the peptide mixture prior to mass spectrometric analysis. A column consisting of Poros reverse phase R2 material (20-30 $\mu \mathrm{m}$ bead size, PerSeptive Biosystems, Foster City, CA) was packed in a constricted GELoader tip (Eppendorf, Hamburg, Germany). Ten microliters of the peptide mixture from the digestion supernatant was diluted $30 \mu \mathrm{l}$ in $0.1 \%$ TFA, loaded onto the column, and washed with $30 \mu \mathrm{l}$ of $0.1 \%$ TFA. The peptides were eluted by $0.8 \mu \mathrm{l}$ of matrix solution $(70 \%$ acetonitrile, $0.1 \%$ TFA, $10 \mathrm{mg} / \mathrm{ml}$ alpha-cyano-4-hydroxycinamic acid). The eluted peptides were spotted onto a stainless steel target plate. Masses of peptides were determined using MALDITOF mass spectrometer (Model M@LDI-R; Micromass, Manchester, UK). Calibration was performed using internal mass of trypsin. Peptide masses were matched with the theoretical peptides of all proteins in the NCBI database using Mascot search program (www.matrixscience.com).

\section{Statistical analysis}

Data were expressed as means \pm S.E.M. Statistical comparisons were performed by one-way analysis of variance and Student's $t$-test. Differences were considered significant at $P<0.05$.

\section{Supplemental data}

Supplemental data include five figures and can be found with this article online at http://e-emm.or.kr/article/article_files/ SP-43-4-02.pdf.

\section{Acknowledgements}

This study was supported by a grant of the Korea Healthcare technology R\&D Project, Ministry for Health \& Welfare, Republic of Korea (No. A000385 \& A030003).

\section{References}

Agirbasli M. Pivotal role of plasminogen-activator inhibitor 1 in vascular disease. Int J Clin Pract 2005;59:102-6

Barrientos S, Stojadinovic O, Golinko MS, Brem $\mathrm{H}$, Tomic-Canic M. Growth factors and cytokines in wound healing. Wound Repair Regen 2008;16:585-601

Binder BR, Mihaly J, Prager GW. UPAR-uPA-PAI-1 interactions and signaling: a vascular biologist's view. Thromb Haemost 2007;97:336-42

Blackburn JS, Brinckerhoff CE. Matrix metalloproteinase-1 and thrombin differentially activate gene expression in endothelial cells via PAR-1 and promote angiogenesis. Am J Pathol 2008;173:1736-46

Chen B, Qin J, Wang H, Magdalou J, Chen L. Effects of adenovirus-mediated bFGF, IL-1Ra and IGF-1 gene transfer on human osteoarthritic chondrocytes and osteoarthritis in rabbits. Exp Mol Med 2010;42:684-95

Chen L, Endler A, Shibasaki F. Hypoxia and angiogenesis: regulation of hypoxia-inducible factors via novel binding factors. Exp Mol Med 2009;41:849-57

Collette J, Bocock JP, Ahn K, Chapman RL, Godbold G, Yeyeodu S, Erickson $\mathrm{AH}$. Biosynthesis and alternate 
targeting of the lysosomal cysteine protease cathepsin L. Int Rev Cytol 2004;241:1-51

D'Alessio S, Blasi F. The urokinase receptor as an entertainer of signal transduction. Front Biosci 2009;14:4575-87

Devy L, Blacher S, Grignet-Debrus C, Bajou K, Masson V, Gerard RD, Gils A, Carmeliet G, Carmeliet P, Declerck PJ, Nöel A, Foidart JM. The pro- or antiangiogenic effect of plasminogen activator inhibitor 1 is dose dependent. FASEB J 2002;16:147-54

Felbor U, Dreier L, Bryant RA, Ploegh HL, Olsen BR, Mothes $W$. Secreted cathepsin $L$ generates endostatin from collagen XVIII. EMBO J 2000;19:1187-94

Fujii T, Yonemitsu Y, Onimaru M, Inoue M, Hasegawa M, Kuwano $H$, Sueishi K. VEGF function for upregulation of endogenous PIGF expression during FGF-2-mediated therapeutic angiogenesis. Atherosclerosis 2008;200:51-7

Gocheva V, Joyce JA. Cysteine cathepsins and the cutting edge of cancer invasion. Cell Cycle 2007;6:60-4

Kaneko T, Fujii S, Matsumoto A, Goto D, Ishimori N, Watano K, Furumoto T, Sugawara T, Sobel BE, Kitabatake A. Induction of plasminogen activator inhibitor-1 in endothelial cells by basic fibroblast growth factor and its modulation by fibric acid. Arterioscler Thromb Vasc Biol 2002;22:855-60

Kim JW, Kim TD, Hong BS, Kim OY, Yoon WH, Chae CB, Gho YS. A serum-stable branched dimeric anti-VEGF peptide blocks tumor growth via anti-angiogenic activity. Exp Mol Med 2010;42:514-23

Kondoh K, Koyama H, Miyata T, Takato T, Hamada H, Shigematsu $\mathrm{H}$. Conduction performance of collateral vessels induced by vascular endothelial growth factor or basic fibroblast growth factor. Cardiovasc Res 2004;61: $132-42$

Lah TT, Durán Alonso MB, Van Noorden CJ. Antiprotease therapy in cancer: hot or not? Expert Opin Biol Ther 2006;6:257-79

Lamalice L, Le Boeuf F, Huot J. Endothelial cell migration during angiogenesis. Circ Res 2007;100:782-94

Le NT, Xue M, Castelnoble LA, Jackson CJ. The dual personalities of matrix metalloproteinases in inflammation. Front Biosci 2007;12:1475-87

Markkanen JE, Rissanen TT, Kivela A, Yla-Herttuala S. Growth factor-induced therapeutic angiogenesis and arteriogenesis in the heart-gene therapy. Cardiovasc Res 2005;65:656-64

Nicholl SM, Roztocil E, Davies MG. Plasminogen activator system and vascular disease. Curr Vasc Pharmacol 2006;4:101-16

Ninomiya M, Koyama H, Miyata T, Hamada H, Miyatake S, Shigematsu $\mathrm{H}$, Takamoto $\mathrm{S}$. Ex vivo gene transfer of basic fibroblast growth factor improves cardiac function and blood flow in a swine chronic myocardial ischemia model. Gene Ther 2003;10:1152-60

Nishikage S, Koyama $\mathrm{H}$, Miyata T, Ishii S, Hamada $\mathrm{H}$, Shigematsu $\mathrm{H}$. In vivo electroporation enhances plasmidbased gene transfer of basic fibroblast growth factor for the treatment of ischemic limb. J Surg Res 2004;120:37-46

Onimaru M, Yonemitsu Y, Tanii M, Nakagawa K, Masaki I, Okano S, Ishibashi H, Shirasuna K, Hasegawa M, Sueishi K. Fibroblast growth factor-2 gene transfer can stimulate hepatocyte growth factor expression irrespective of hypoxiamediated downregulation in ischemic limbs. Circ Res 2002; 91:923-30

Palermo C, Joyce JA. Cysteine cathepsin proteases as pharmacological targets in cancer. Trends Pharmacol Sci 2008;29:22-8

Palmon A, Roos H, Edel J, Zax B, Savion N, Grosskop A, Pitaru S. Inverse dose- and time-dependent effect of basic fibroblast growth factor on the gene expression of collagen type I and matrix metalloproteinase- 1 by periodontal ligament cells in culture. J Periodontol 2000;71:974-80

Pardo A, Selman M. MMP-1: the elder of the family. Int $\mathrm{J}$ Biochem Cell Biol 2005;37:283-8

Pintucci G, Moscatelli D, Saponara F, Biernacki PR, Baumann FG, Bizekis C, Galloway AC, Basilico C, Mignatti P. Lack of ERK activation and cell migration in FGF-2deficient endothelial cells. FASEB J 2002;16:598-600

Pulukuri SM, Rao JS. Matrix metalloproteinase-1 promotes prostate tumor growth and metastasis. Int J Oncol 2008; 32:757-65

Skrzydlewska E, Sulkowska M, Koda M, Sulkowski S. Proteolytic-antiproteolytic balance and its regulation in carcinogenesis. World J Gastroenterol 2005;11:1251-66

Stoka V, Turk V, Turk B. Lysosomal cysteine cathepsins: signaling pathways in apoptosis. Biol Chem 2007;388: $555-60$

Turner N, Grose R. Fibroblast growth factor signalling: from development to cancer. Nat Rev Cancer 2010;10:116-29

Urbich C, Heeschen C, Aicher A, Sasaki K, Bruhl T, Farhadi MR, Vajkoczy P, Hofmann WK, Peters C, Pennacchio LA, Abolmaali ND, Chavakis E, Reinheckel T, Zeiher AM, Dimmeler $S$. Cathepsin $L$ is required for endothelial progenitor cell-induced neovascularization. Nat Med 2005;11: 206-13

van Hinsbergh VW, Engelse MA, Quax PH. Pericellular proteases in angiogenesis and vasculogenesis. Arterioscler Thromb Vasc Biol 2006;26:716-28

Watanabe A, Kurabayashi M, Arai M, Sekiguchi K, Nagai R. Combined effect of retinoic acid and basic FGF on PAI-1 gene expression in vascular smooth muscle cells. Cardiovasc Res 2001;51:151-9

Xie J, Bian H, Qi S, Xu Y, Tang J, Li T, Liu X. Effects of basic fibroblast growth factor on the expression of extracellular matrix and matrix metalloproteinase-1 in wound healing. Clin Exp Dermatol 2008;33:176-82

Yang Z, Cox JL. Cathepsin L increases invasion and migration of B16 melanoma. Cancer Cell Int 2007;7:8

Yla-Herttuala S. An update on angiogenic gene therapy: vascular endothelial growth factor and other directions. Curr Opin Mol Ther 2006;8:295-300 